\title{
Methodological Unification of the Real-time Algorithm for Wave Generation in a Test Basin and Consideration of its Calculation Error
}

\author{
by Ben T. Nohara, Member
}

\begin{abstract}
Summary
This paper describes the real-time algorithm of ocean waves generated by the snake type wave maker in a test basin. The developed real-time algorithm forms a unified computation process against various kinds of waves. Moreover, a lattice filter like structure of this algorithm is shown.

The algorithm takes an iterative formulation on its calculation process. An iterative calculation generally has a progressive error when the number of iteration becomes large. However, the algorithm causes only a little error after the one million times iteration. Further, the spectrum profiles generated by this method and the conventional one are discussed.
\end{abstract}

\section{Introduction}

The important of model testing using a wave maker is on the increase. Simulation of real ocean condition in the laboratory is indispensable to overcome the design limitations.

The author has developed the real-time algorithm which generates the multi-directional waves ${ }^{1 / 2)}$. However, the former studies have been lacking in consideration of the calculation error depending on the iterative process. The error caused by the iterative process has not been cleared. The algorithm structure also has been in short consideration.

The following section summarizes several waves generated by the snake type wave maker and its mathematical formulae. Section 3 deals with the real-time algorithm of the wave synthesis corresponding to section 2. Section 4 discusses the error caused by the real -time calculation as well as the structure of the realtime algorithm. The final section describes the concluding remarks.

\section{Ocean Waves and Generation Formulae in the Snake Type Wave Maker}

A fundamental wave of ocean waves is formulated as a sinusoidal function, which is based on the potential wave theory ${ }^{3)}$. Therefore various kinds of ocean waves can be written as a linear superposition of a large number of sinusoidal waves. Above all, so-called short

Mitsubishi Heavy Industries, Ltd.

Received 9th July 1997

Read at the Autumn meeting 14, 15th Nov. 1997 crested waves are multi-directional irregular waves all traveling independently of one another in different directions with different frequencies.

The model test using multi-directional irregular waves is in the process of being a main current. However, other several waves must be prepared to obtain the basic data.

\subsection{Regular waves}

The regular wave is the artificial but fundamental wave for obtaining the basic data acting on the test body. The regular wave with its propagation direction of $\theta$ is generated by the following wave paddle motion. The wave paddle motion $\eta_{i}(t)$ of the $i$-th segmented wave paddle at time $t$ can be formulated as

$$
\eta_{i}(t)=\frac{a}{2 F} \sin (\sigma t-i k b \cos \theta)
$$

where $a, \sigma, b$ and $t$ denote amplitude, angular frequency, wave paddle width and time, respectively, and $F$ indicates transfer function for wave generation. $F$ is represented by

$$
F=\frac{4 \sinh (k h)}{k h} \frac{1-\cosh (k h)+(k h) \sinh (k h)}{2 k h+\sinh (2 k h)}
$$

for flap type wave paddle ${ }^{4)}$, where $h$ means the uniform water depth in the basin. Moreover, $k$ is wave number and a solution of the dispersion relation as follows.

$$
\sigma^{2}=k g \tanh (k h)
$$

Here $g$ indicates the acceleration due to gravity.

\subsection{Long-crested irregular waves}

The long-crested irregular wave has its original spectrum such as ISSC $^{5)}$, JONSWAP ${ }^{6}$, and BretschneiderMitsuyasu $^{7}$ etc., therefore can be considered as a linear superposition of a large number of sinusoidal waves with different frequencies. However this wave also progresses toward a direction same as the regular wave. 
In the same manner as the regular wave, the flap motion $\eta_{i}(t)$ can be formulated as

$$
\eta_{i}(t)=\sum_{n=1}^{N} \frac{a_{n}}{F_{n}} \sin \left(\sigma_{n} t-i k_{n} b \cos \theta+\varepsilon_{n}\right)
$$

where $N, \sigma_{n}, k_{n}, \varepsilon_{n}$ and $F_{n}$ are the number of component waves in frequency, the angular frequency of $n$-th component wave, the wave number of $n$-th component wave, the random phase lag defined from 0 to $2 \pi$ of $n^{-}$ th component wave and the transfer function of $n$-th component wave, respectively. $F_{n}$ is represented by the following equation for flap type wave paddle ${ }^{4}$.

$$
F_{n}=\frac{4 \sinh \left(k_{n} h\right)}{k_{n} h} \frac{1-\cosh \left(k_{n} h\right)+\left(k_{n} h\right) \sinh \left(k_{n} h\right)}{2 k_{n} h+\sinh \left(2 k_{n} h\right)}
$$

Here $k_{n}$ is obtained by

$$
\sigma_{n}{ }^{2}=k_{n} g \tanh \left(k_{n} h\right) \text {. }
$$

Moreover, $a_{n}$ is the amplitude of $n$-th component wave and represented by

$$
a_{n}=\sqrt{2 S\left(\sigma_{n}\right) \Delta \sigma_{n}},
$$

where $S$ denotes the frequency spectrum and $\Delta \sigma_{n}$ represents the minute spectrum bandwidth.

\section{3 Multi-directional irregular waves}

This wave is the so-called short crested wave which has a number of directional components added to the frequency components of the irregular wave. To generate this wave, the wave paddle motion $\eta_{i}(t)$ can be formulated by the double summation model $^{8)}$ as

$$
\eta_{i}(t)=\sum_{n=1}^{N} \sum_{m=1}^{M} \frac{a_{n m}}{F_{n}} \sin \left(\sigma_{n} t-i k_{n} b \cos \theta_{m}+\varepsilon_{n m}\right)
$$

where $M, a_{n m}, \theta_{m}$, and $\varepsilon_{n m}$ are the number of component waves in direction, the amplitude of $n \mathrm{~m}$-th component wave, the $m$-th propagation direction, and the random phase lag defined from 0 to $2 \pi$ of $n m$-th component wave, respectively. The amplitude of $n m$-th component wave, $a_{n m}$, is represented by

$$
a_{n m}=\sqrt{2 S_{\eta}\left(\sigma_{n}, \theta_{m}\right) \Delta \sigma_{n} \Delta \theta_{m}},
$$

where $\Delta \theta_{m}$ represents the directional resolution and $S_{\eta}$ denotes directional spectrum which is given by

$$
S_{\eta}(\sigma, \theta)=S(\sigma) G(\theta) \text {. }
$$

$G(\theta)$ denotes directional distribution function which is represented by ${ }^{9)}$

$$
\begin{aligned}
& G(\theta)=\frac{\Gamma(\gamma+1)}{\sqrt{\pi} \Gamma\left(\gamma+\frac{1}{2}\right)} \cos ^{2 \gamma}\left(\theta-\theta_{0}\right), 0 \leqq \theta \leqq \pi, \\
& \gamma=1,2,3, \cdots
\end{aligned}
$$

where $\Gamma, \gamma$ and $\theta_{0}$ mean Gamma function, the index number of the directional concentration and main wave stream line, respectively.

\section{4 Complex waves}

This wave is defined by the summation of longcrested irregular waves and short-crested irregular waves. This wave corresponds to the situation: the wind waves are piled up the swell. The wind waves are normally short-crested irregular waves with short wavelength and created near by the current position and the swell is created at far away and becomes only low frequency (long wavelength) components as pro- gressing with vanishing high frequency component waves.

The wave paddle motion $\eta_{i}(t)$ for the complex wave can be formulated as the summation of some regular waves and irregular waves, therefore mathematically written by as follows from equation (1) and equation (4):

$$
\begin{aligned}
\eta_{i}(t)= & \sum_{n=1}^{\hat{N}} \frac{\hat{a}_{n}}{F_{n}} \sin \left(\widehat{\sigma}_{n} t-i \widehat{k}_{n} b \cos \theta+\widehat{\varepsilon}_{n}\right) \\
& +\sum_{n=1}^{\bar{N}} \sum_{m=1}^{\bar{M}} \frac{\tilde{a}_{n m}}{F_{n}} \sin \left(\tilde{\sigma}_{n} t-i \tilde{k}_{n} b \cos \tilde{\theta}_{m}+\tilde{\varepsilon}_{n m}\right)
\end{aligned}
$$

In this equation, the circumflex and - means parameters of the long-crested irregular wave and the shortcrested irregular wave, respectively. Each suffix of $n$ and $m$ must be carefully read.

\section{Real-time Algorithm ${ }^{10)}$}

Calculation of generation signal of waves, especially multi-directional irregular waves and/or complex waves, is troublesome work with the evaluation of many trigonometric functions. To complete the computation within a time-step of the wave generation is too hard even by a currently modernized computer. Therefore wave generation signals for all wave maker units usually have to be calculated beforehand. That is, an operator of a wave maker has to wait a long stand-by time to compute generation signals defined by a next wave condition, because the preparation of calculation irregular wave generation needs a much longer computation time than the duration of actual wave generation $^{11}$. To eliminate the problem described above, the author has formulated and employed the iterative computation method for the generation of various kinds of waves in real-time. Moreover, the unified algorithm for the generation of various kinds of waves is induced.

\section{1 Regular waves}

Let equation (1) be changed to the discrete time equation as follows:

$$
\eta_{i j}=\frac{a}{2 F} \sin (j \sigma \Delta t-i k b \cos \theta),
$$

where $j$ denotes the $j$-th time step.

Here

$$
\begin{aligned}
& \xi_{i}=\frac{a}{2 F} \sin (-i k b \cos \theta) \\
& \zeta_{i}=\frac{a}{2 F} \cos (-i k b \cos \theta),
\end{aligned}
$$

then

$$
\eta_{i j}=\xi_{i} \cos (j \sigma \Delta t)+\zeta_{i} \sin (j \sigma \Delta t) .
$$

Now let $\varphi_{i j}$ and $\Phi_{i j}$ be

$$
\left(\begin{array}{c}
\varphi_{i j} \\
\Phi_{i j}
\end{array}\right)=\left(\begin{array}{cc}
\xi_{i} & \zeta_{i} \\
\zeta_{i} & -\xi_{i}
\end{array}\right)\left(\begin{array}{l}
\cos (j \sigma \Delta t) \\
\sin (j \sigma \Delta t)
\end{array}\right),
$$

then the following iterative equation is obtained. That is,

$$
\left(\begin{array}{c}
\varphi_{i, j+1} \\
\Phi_{i, j+1}
\end{array}\right)=\left(\begin{array}{cc}
\alpha & \beta \\
-\beta & \alpha
\end{array}\right)\left(\begin{array}{c}
\varphi_{i j} \\
\Phi_{i j}
\end{array}\right)
$$


where

$$
\begin{aligned}
& \alpha=\cos (\sigma \Delta t) \\
& \beta=\sin (\sigma \Delta t) .
\end{aligned}
$$

As the results of that, by calculating equations (14), (15), (19), and (20) in advance, $\varphi_{i, j+1}$, i. e., $\eta_{i, j+1}$ is obtained by equation (18) without sinusoidal calculation.

\section{2 Irregular waves}

In the case of the irregular wave, similarly, the followings are shown.

Let equation (4) be changed to the discrete time equation as follows:

$$
\eta_{i j}=\sum_{n=1}^{N} \frac{a_{n}}{F_{n}} \sin \left(j \sigma_{n} \Delta t-i k_{n} b \cos \theta+\varepsilon_{n}\right)
$$

Here

$$
\begin{aligned}
& \xi_{n i}=\frac{a_{n}}{F_{n}} \sin \left(-i k_{n} b \cos \theta+\varepsilon_{n}\right) \\
& \zeta_{n i}=\frac{a_{n}}{F_{n}} \cos \left(-i k_{n} b \cos \theta+\varepsilon_{n}\right),
\end{aligned}
$$

then

$$
\eta_{i j}=\sum_{n=1}^{N}\left\{\xi_{n i} \cos \left(j \sigma_{n} \Delta t\right)+\zeta_{n i} \sin \left(j \sigma_{n} \Delta t\right)\right\} .
$$

Now let $\varphi_{n i j}$ and $\Phi_{n i j}$ be

$$
\left(\begin{array}{c}
\varphi_{n i j} \\
\Phi_{n i j}
\end{array}\right)=\left(\begin{array}{cc}
\xi_{n i} & \zeta_{n i} \\
\zeta_{n i} & -\xi_{n i}
\end{array}\right)\left(\begin{array}{l}
\cos \left(j \sigma_{n} \Delta t\right) \\
\sin \left(j \sigma_{n} \Delta t\right)
\end{array}\right)
$$

then the following iterative equation is obtained. That is,

$$
\left(\begin{array}{c}
\varphi_{n i, j+1} \\
\Phi_{n i, j+1}
\end{array}\right)=\left(\begin{array}{cc}
\alpha_{n} & \beta_{n} \\
-\beta_{n} & \alpha_{n}
\end{array}\right)\left(\begin{array}{c}
\varphi_{n i j} \\
\Phi_{n i j}
\end{array}\right)
$$

where

$$
\begin{aligned}
& \alpha_{n}=\cos \left(\sigma_{n} \Delta t\right) \\
& \beta_{n}=\sin \left(\sigma_{n} \Delta t\right) .
\end{aligned}
$$

By calculating equations (22), (23), (27), and (28) in advance, $\varphi_{i, j+1}$ is obtained by equation (26) without sinusoidal calculation.

Finally, $\eta_{i, j+1}$ is obtained by equation (24) as follows:

$$
\eta_{i, j+1}=\sum_{n=1}^{N} \varphi_{n i, j+1} \text {. }
$$

\section{3 Multi-directional irregular waves}

The case of the multi-directional irregular wave is shown similarly.

Let equation ( 8 ) be changed to the discrete time equation as follows:

$$
\eta_{i j}=\sum_{n=1}^{N} \sum_{m=1}^{M} \frac{a_{n m}}{F_{n}} \sin \left(j \sigma_{n} \Delta t-i k_{n} b \cos \theta_{m}+\varepsilon_{n m}\right)
$$

Here

$$
\begin{aligned}
& \xi_{n i}=\sum_{m=1}^{M} \frac{a_{n m}}{F_{n}} \sin \left(-i k_{n} b \cos \theta_{m}+\varepsilon_{n m}\right) \\
& \zeta_{n i}=\sum_{m=1}^{M} \frac{a_{n m}}{F_{n}} \cos \left(-i k_{n} b \cos \theta_{m}+\varepsilon_{n m}\right),
\end{aligned}
$$

then equation (24) is obtained.

Now let $\varphi_{n i j}$ and $\Phi_{n i j}$ be equation (25). Then equation (26) and equation (29) are obtained.

\section{4 Complex waves}

The calculation procedure is similar, therefore only results are shown.

The discrete time equation:

$$
\eta_{i j}=\sum_{n=1}^{\bar{N}} \frac{\widehat{a}_{n}}{F_{n}} \sin \left(j \widehat{\sigma}_{n} \Delta t-i \widehat{k}_{n} b \cos \theta+\widehat{\varepsilon}_{n}\right)
$$

$$
+\sum_{n=1}^{\tilde{N}} \sum_{m=1}^{\tilde{M}} \frac{\tilde{a}_{n m}}{F_{n}} \sin \left(j \tilde{\sigma}_{n} \Delta t-i \tilde{k}_{n} b \cos \tilde{\theta}_{m}+\tilde{\varepsilon}_{n m}\right)
$$

Parameters :

$$
\begin{aligned}
\xi_{n i}= & {\left[\begin{array}{ll}
\widehat{\xi}_{n i} & \tilde{\xi}_{n i}
\end{array}\right]=\left[\frac{\widehat{a}_{n}}{F_{n}} \sin \left(-i \widehat{k}_{n} b \cos \theta+\widehat{\varepsilon}_{n}\right)\right.} \\
& \left.\sum_{m=1}^{\bar{M}} \frac{\tilde{a}_{n m}}{F_{n}} \sin \left(-i \widetilde{k}_{n} b \cos \tilde{\theta}_{m}+\widetilde{\varepsilon}_{n m}\right)\right] \\
\zeta_{n i}= & {\left[\begin{array}{ll}
\hat{\zeta}_{n i} & \tilde{\zeta}_{n i}
\end{array}\right]=\left[\frac{\hat{a}_{n}}{F_{n}} \cos \left(-i \widehat{k}_{n} b \cos \theta+\widehat{\varepsilon}_{n}\right)\right.} \\
& \left.\sum_{m=1}^{\tilde{M}} \frac{\tilde{a}_{n m}}{F_{n}} \cos \left(-i \tilde{k}_{n} b \cos \tilde{\theta}_{m}+\widetilde{\varepsilon}_{n m}\right)\right]
\end{aligned}
$$

The equation which is rewritten by using equation (34) and equation (35) in equation (33):

$$
\eta_{i j}=\sum_{n=1}^{\bar{N}}(1 \quad 1) \varphi_{n i j}, \bar{N}=\max (\hat{N}, \tilde{N})
$$

where $\varphi_{n i j}, \Phi_{n i j}$ :

$$
\begin{aligned}
& \varphi_{n i j}=\left[\begin{array}{ll}
\widehat{\varphi}_{n i j} & \tilde{\varphi}_{n i j}
\end{array}\right]^{T} \\
& \Phi_{n i j}=\left[\begin{array}{ll}
\widehat{\Phi}_{n i j} & \widetilde{\Phi}_{n i j}
\end{array}\right]^{T} \\
& \left(\begin{array}{c}
\widehat{\varphi}_{n i j} \\
\widehat{\Phi}_{n i j}
\end{array}\right)=\left(\begin{array}{cc}
\widehat{\xi}_{n i} & \bar{\zeta}_{n i} \\
\widetilde{\zeta}_{n i} & -\bar{\xi}_{n i}
\end{array}\right)\left(\begin{array}{l}
\cos \left(j \widehat{\sigma}_{n} \Delta t\right) \\
\sin \left(j \widehat{\sigma}_{n} \Delta t\right)
\end{array}\right) \\
& \left(\begin{array}{c}
\widetilde{\varphi}_{n i j} \\
\widetilde{\Phi}_{n i j}
\end{array}\right)=\left(\begin{array}{cc}
\widetilde{\xi}_{n i} & \widetilde{\zeta}_{n i} \\
\widetilde{\zeta}_{n i} & -\widetilde{\xi}_{n i}
\end{array}\right)\left(\begin{array}{l}
\cos \left(j \widetilde{\sigma}_{n} \Delta t\right) \\
\sin \left(j \widetilde{\sigma}_{n} \Delta t\right)
\end{array}\right)
\end{aligned}
$$

The iterative equation:

$$
\left(\begin{array}{c}
\varphi_{n i, j+1} \\
\Phi_{n i, j+1}
\end{array}\right)=\left(\begin{array}{cc}
\alpha_{n} & \beta_{n} \\
-\beta_{n} & \alpha_{n}
\end{array}\right)\left(\begin{array}{c}
\varphi_{n i j} \\
\Phi_{n i j}
\end{array}\right)
$$

where

$$
\begin{aligned}
& \alpha_{n}=\operatorname{diag}\left(\begin{array}{ll}
\hat{\alpha}_{n} & \tilde{\alpha}_{n}
\end{array}\right) \\
& \beta_{n}=\operatorname{diag}\left(\begin{array}{ll}
\bar{\beta}_{n} & \tilde{\beta}_{n}
\end{array}\right) \\
& \widehat{\alpha}_{n}=\cos \left(\widehat{\sigma}_{n} \Delta t\right) \\
& \tilde{\alpha}_{n}=\cos \left(\tilde{\sigma}_{n} \Delta t\right) \\
& \bar{\beta}_{n}=\sin \left(\widehat{\sigma}_{n} \Delta t\right) \\
& \widetilde{\beta}_{n}=\sin \left(\widetilde{\sigma}_{n} \Delta t\right) \text {. }
\end{aligned}
$$

\section{Consideration of the Calculation Error}

\section{1 Structure of the calculation}

The structure of the iterative equations (18), (26) and (41) is considered here. The matrixes of coefficients of equations (18), (26) and (41) are the orthogonal matrixes which represent rotation. So, $\varphi^{*}$ and $\Phi^{*}$ are guaranteed to be stable numerically depending on this calculation process.

Figure 1 shows the structure of equations (18), (26) and (41) by expressing in the digital filters. This structure is similar to a lattice filter in the field of signal processing $^{12)}$.

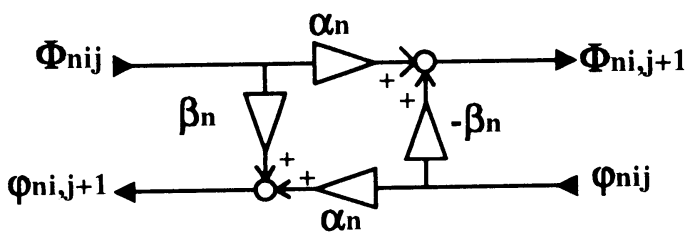

Fig. 1 Structure of equations (18), (26) and (41): a lattice filter like structure. 


\section{2 Calculation error}

First of all, let's consider the calculation error when the iteration process is executed. A simple example is as follows:

The direct calculation is $b / a$, where both $a$ and $b$ are integers. The iterative process to obtain $b / a$ is defined as the function $f_{n}(a, b)$

$$
f_{n}(a, b)=\frac{b}{c_{1}} \frac{c_{1}}{c_{2}} \frac{c_{2}}{c_{3}} \ldots \frac{c_{i}}{c_{i+1}} \ldots \frac{c_{n}}{a} .
$$

Here, $c^{*}$ and $n$ are uniform random integer values and integer, respectively.

$f_{n}(a, b)$ equals $b / a$ theoretically. However, the error $e$ :

$$
e=\left|\frac{b}{a}-f_{n}(a, b)\right|
$$

becomes progressively larger when $\mathrm{n}$ becomes large. That is, the value of the error $e$ depends on the value of $n$. Figure 2 shows the state of the increasing error as the iteration.

The calculation error of the real-time algorithm described in section 3 is considered next. In the case of the multi-directional irregular wave, the calculation error $e_{w}$ is defined by the following equation.

$$
\begin{aligned}
e_{w}= & \sum_{n=1}^{N} \sum_{m=1}^{M} \frac{a_{n m}}{F_{n}} \sin \left(j \sigma_{n} \Delta t-i k_{n} b \cos \theta_{m}+\varepsilon_{n m}\right) \\
& -\sum_{n=1}^{N}\left(\begin{array}{cc}
1 & 0
\end{array}\right) \underbrace{\left(\begin{array}{cc}
\alpha_{n} & \beta_{n} \\
-\beta_{n} & \alpha_{n}
\end{array}\right)\left(\begin{array}{cc}
\alpha_{n} & \beta_{n} \\
-\beta_{n} & \alpha_{n}
\end{array}\right) \cdots\left(\begin{array}{cc}
\alpha_{n} & \beta_{n} \\
-\beta_{n} & \alpha_{n}
\end{array}\right)}_{j \text { times }} \\
& \times\left(\begin{array}{l}
\sum_{m=1}^{M} \frac{a_{n m}}{F_{n}} \sin \left(-i k_{n} b \cos \theta_{m}+\varepsilon_{n m}\right) \\
\sum_{m=1}^{M} \frac{a_{n m}}{F_{n}} \cos \left(-i k_{n} b \cos \theta_{m}+\varepsilon_{n m}\right)
\end{array}\right)
\end{aligned}
$$

Figure 3 shows the time histories from 990,620 to 1,003 , 000 in the sampling number generated by the direct method: equation (30) with the parameters: $M=70$, $N=200, b=0.4[\mathrm{~m}], \Delta t=4[\mathrm{msec}], H_{1 / 3}=0.3[\mathrm{~m}], T_{01}=0$. $75[\mathrm{sec}]$ and the generated spectrum: ISSC. Figure 4 shows the same one, but generated by the iterative method. Figure 5 shows the error defined by equation (50). The arithmetic calculation is executed by the double precision floating point. Figure 5 indicates that this iterative method causes only the error of the order of $10^{-3}$ after the one million times iteration.

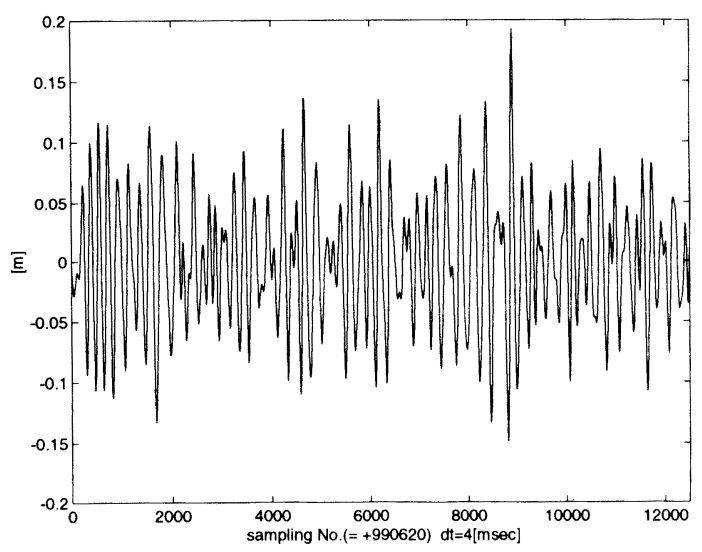

Fig. 3 Generated waves by equation (30) : the direct method.

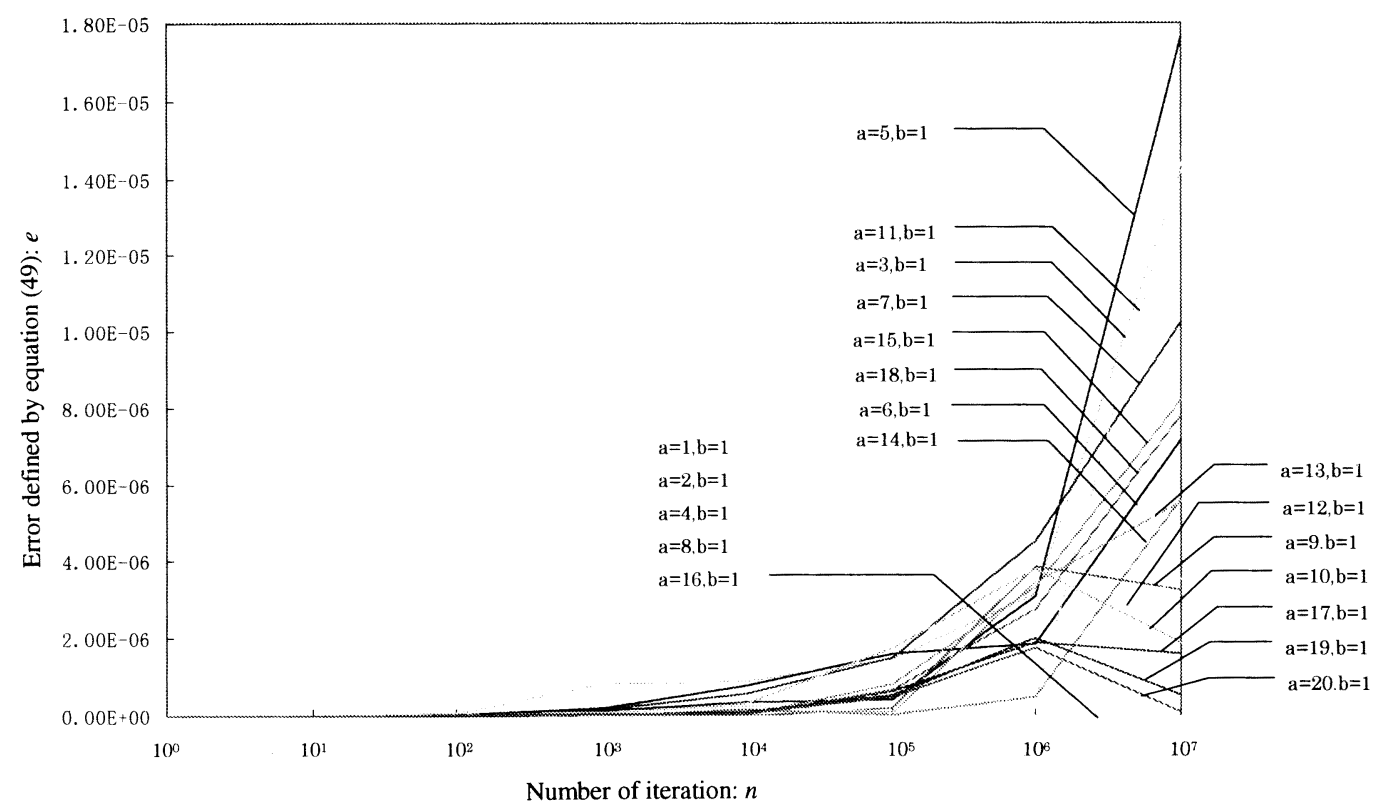

Fig. 2 State of the increasing error. 


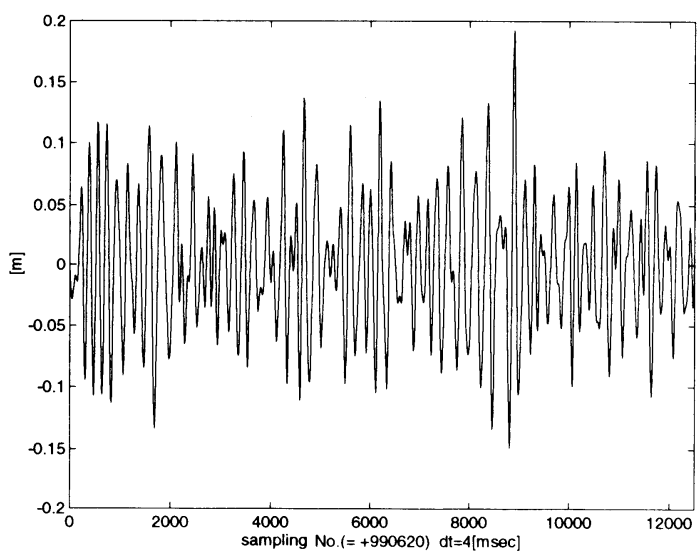

Fig. 4 Generated waves by the iterative algorithm.

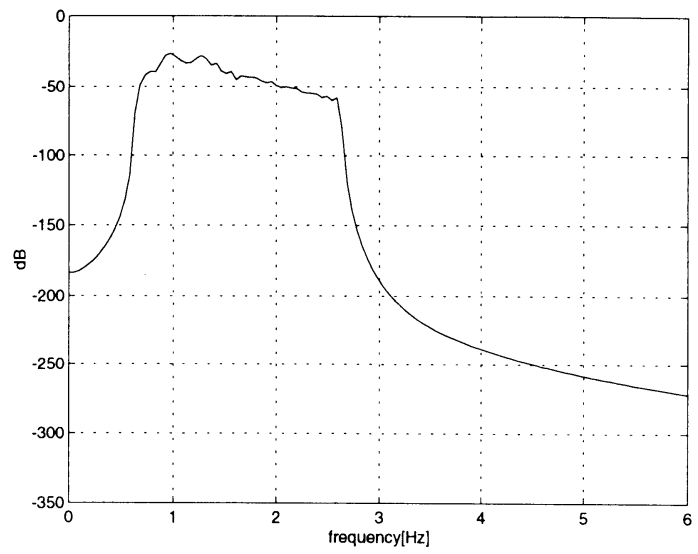

(a) Spectrum profile obtained from figure 3 .

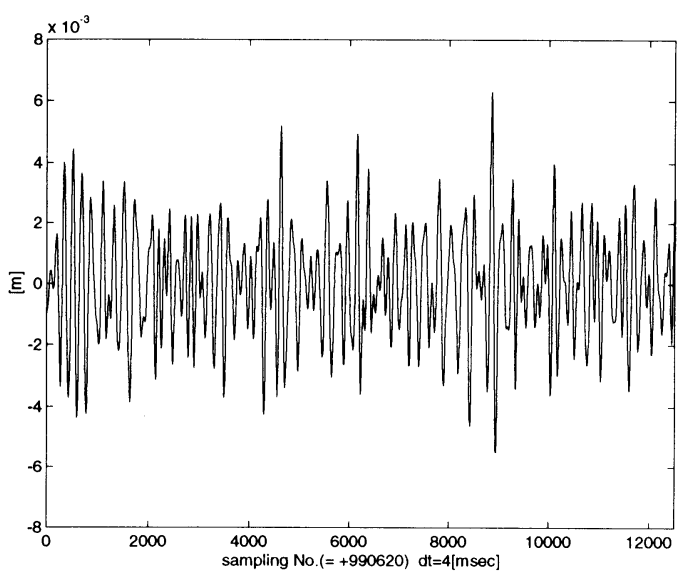

Fig. 5 Calculation error between the direct method and the iterative algorithm defined by equation (50).

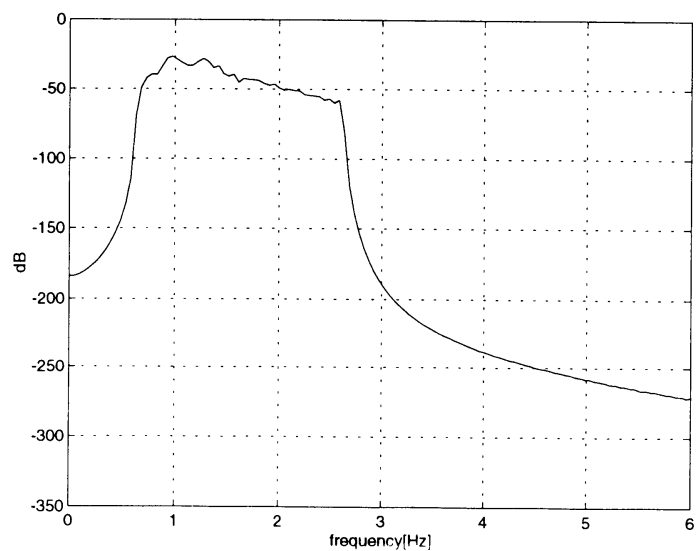

(b) Spectrum profile obtained from figure 4.

Fig. 6 Power spectrum : 512-point-FFT, 90\% overlapping, 25 times averaging.

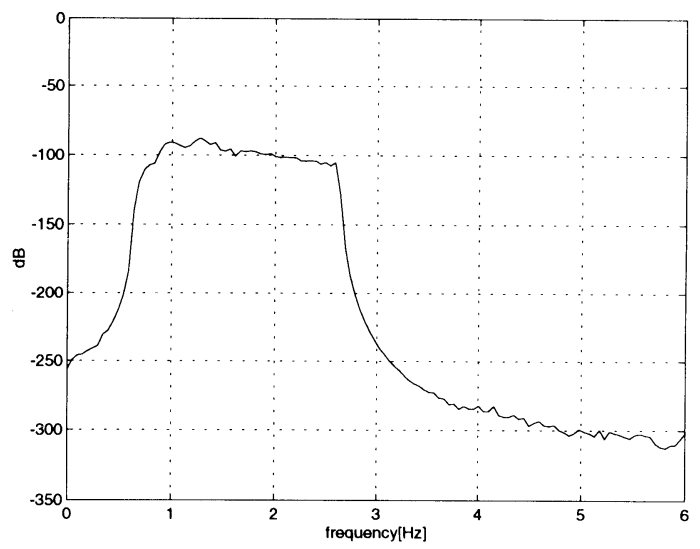

Fig. 7 Power spectrum of the calculation error.
The spectrum profile is more important rather than the absolute accuracy in the model test using a wave maker. So, the next is the discussion concerning the spectrum profile comparison between the direct method and the iterative method. Figure 6 shows the power spectrum obtained from Figure 3 and Figure 4. These power spectra are calculated by FFT algorithm indicated in the figure's caption. These profiles are in good agreement. Moreover, Figure 7 shows the power spectrum of the error : Figure 5. The error spectrum is very low $(-100[\mathrm{~dB}])$, so the iterative error has no effect on the spectrum profile of the iterative method.

\section{Concluding Remark}

The author has reached a methodological unification of the real-time algorithm for wave generation in a test basin. The structure of this algorithm has the orth- 
ogonal matrix which represents rotation and is similar to a lattice filter.

The algorithm causes only the error of the order of $10^{-3}$ after the one million times iteration which equals 66.7 minutes. Moreover, the spectrum profile of the algorithm gives good agreement with the one which is obtained by the direct method.

\section{References}

1) Nohara, B. T., Yamamoto, I., and Matsuura, M., "Multi-directional Wave Maker and its Real Time Wave Control System Applied to a Seakeeping Model Basin," Wave Generation '95, Yokohama, Japan, (1995) pp. 175-194.

2) Nohara, B. T., Yamamoto, I., and Matsuura, M., "Development of Real Time Wave Simulation Technique," Journal of Soc. of Naval Arch. of Japan, Vol. 180, (1996) pp. 159-163.

3) For example, Crapper, G. D., "Introduction to Water Waves," Ellis Horwood Ltd. (1984)

4) Biesel, F. and Suquet, F., "Les apparails generateurs de houle en laboratoire," La Houille Blanche, 6, 2, 4, and 5, (1951)

5) Huang, N. E., Tung, C., and Long, S. R., "Wave Spectra," Ocean Engineering Science, The Sea, Volume 9, Part A, (1990) pp. 197-237.

6) Hasselmann, K. et al., "Measurement of wind- wave growth and swell decay during the Joint North Sea Wave Project (JONSWAP)," Deutsche Hydrograph, Z., A 8 (No. 12), (1973)

7) Mitsuyasu, W., "Development of Spectra of Wind and Waves (2)," in Proceedings of the 17 th Coastal Engineering Conference, (1970) pp. 1-7.

8) Takayama, T. and Hiraishi, T., "Reproducibility of Directional Random Waves in Laboratory Wave Simulation," Report of the Port and Harbour Research Institute, 28, 4, (1989) pp. 3-24

9) Maeda, H., at al., "Application of Walsh Function to Multi-Directional Ocean Wave Simulation," Journal of Soc. of Naval Arch. of Japan, 177, (1995) pp. 187-195 (in Japanese).

10) Nohara, B. T., "Equipment for Vibration Generation," Patent, Application number 7-235709, Japan, (1995) (in Japanese)

11) Matsuura, M., Yamaguchi, Y., Yamamoto, I., and Nohara, B. T., "An Improved Method to Calculate Generation Signal of Multidirectional Irregular Waves," in Proceedings of IAHR Seminar on Multidirectional Waves and Their Interaction with Structures, The 27 th International Association for Hydraulic Research 1997 Congress Conference, San Francisco, USA, (1997) pp. 241-254.

12) For example, Zelniker, G. and Taylor, F. J., "Advanced Digital Signal Processing," Marcel Dekker, (1994) pp. 291-302. 